\title{
Benign pelvic masses masquerading as adnexal cancer during pregnancy on ultrasound: A retrospective study of 5 years
}

\author{
MENG XIE ${ }^{1,2 *}$, XUYIN ZHANG ${ }^{3 *}$, WENPING WANG ${ }^{2}$ and KEQIN HUA ${ }^{3}$ \\ ${ }^{1}$ Department of Ultrasound, Obstetrics and Gynecology Hospital, Fudan University, Shanghai 200090; \\ ${ }^{2}$ Department of Ultrasound, Zhongshan Hospital, Fudan University, Shanghai 200032; ${ }^{3}$ Department of Obstetrics \\ and Gynecology, Obstetrics and Gynecology Hospital, Fudan University, Shanghai 200090, P.R. China
}

Received May 20, 2015; Accepted July 30, 2015

DOI: $10.3892 / \mathrm{mco} .2015 .632$

\begin{abstract}
This study was conducted to investigate the sonographic characteristics of benign adnexal masses misdiagnosed as malignancy, and determine whether sonographic findings may help distinguish these lesions from malignant tumors during pregnancy at a single center. We herein present our experience with pregnant women with benign adnexal masses misdiagnosed as malignancy by ultrasound and operated on during pregnancy or after delivery. A total of 4 cases of benign pelvic masses were misdiagnosed as adnexal cancer over a 5-year period, including an ovarian theca cell tumor with luteinization, an obsolete ectopic pregnancy of fallopian tube, an ovarian mature teratoma with abundant nervous tissue and a uterine myoma with degeneration. The masses were characterized by abundant blood flow and heterogeneous echo on ultrasound. In conclusion, adnexal masses identified during pregnancy exhibiting abundant blood flow and heterogeneous echo may not represent malignancy, even in the presence of increased carbohydrate antigen 125 levels. In certain cases, benign adnexal masses may be initially misdiagnosed as malignant during pregnancy.
\end{abstract}

\section{Introduction}

Malignant adnexal masses are rare during pregnancy. The imaging characteristics of such masses have been even less frequently reported. The sonographic appearance of benign adnexal masses may be misdiagnosed as malignancy and

Correspondence to: Professor Wenping Wang, Department of Ultrasound, Zhongshan Hospital, Fudan University, 180 Fen Lin Road, Shanghai 200032, P.R. China

E-mail: wenpingwang11@163.com

Professor Keqin Hua, Department of Obstetrics and Gynecology, Obstetrics and Gynecology Hospital, Fudan University, 128 Shen Yang Road, Shanghai 200090, P.R. China

E-mail: huakeqinjiaoshou@163.com

${ }^{*}$ Contributed equally

Key words: ovarian cancer, ultrasound, pregnancy further research is required to determine whether sonographic findings may distinguish these lesions from malignant tumors during pregnancy (1-3). The aim of this study was to describe the preoperative sonographic characteristics of 4 consecutive patients with lesions misdiagnosed as malignant and histologically diagnosed as benign postoperatively.

\section{Patients and methods}

Cases. For this retrospective study, we collected cases of pregnancy complicated by adnexal masses treated at the Obstetrics and Gynecology Hospital of Fudan University (Shanghai, China) between January, 2010 and January, 2014. All the patients had undergone imaging using a transabdominal 13-MHz probe and we selected a total of 4 cases with benign pelvic masses initially misdiagnosed as ovarian cancer. This study was approved by the Obstetrics and Gynecology Hospital of Fudan University Institutional Review Board (Shanghai, China).

\section{Results}

Case 1. The patient was admitted at 23 weeks of gestation for a heterogeneous adnexal mass, sized 8x7x6 cm, which was discovered incidentally. Sonographically, there was a predominantly solid lesion with a middle hypoechoic area on the left adnexa, appearing to originate from the left ovary. Color Doppler evaluation (MyLab 90 Systems; Esaote S.p.A., Genova, Italy) demonstrated abundant arterial and venous flow (Fig. 1). We detected an accumulation of 1,000 $\mathrm{ml}$ of ascitic fluid. The fetus and the contralateral adnexa were normal in appearance. The mass was diagnosed as malignant left ovarian tumor on ultrasound. The carbohydrate antigen (CA) 125 levels were increased to $2,443.1 \mathrm{U} / \mathrm{ml}$ (normal, <35 U/ml). During laparoscopy a highly vascularized mass was identified at the periphery of the left ovary. There was a $800-\mathrm{ml}$ blood loss and the patient received a transfusion intraoperatively (Fig. 2). The mass was diagnosed as luteoma of pregnancy on frozen section analysis. However, on postoperative pathological examination, the mass was definitively diagnosed as a theca cell tumor with luteinization. The follow-up of the pregnancy course was unremarkable and the patient delivered a 3,200-g infant at 39 weeks. 


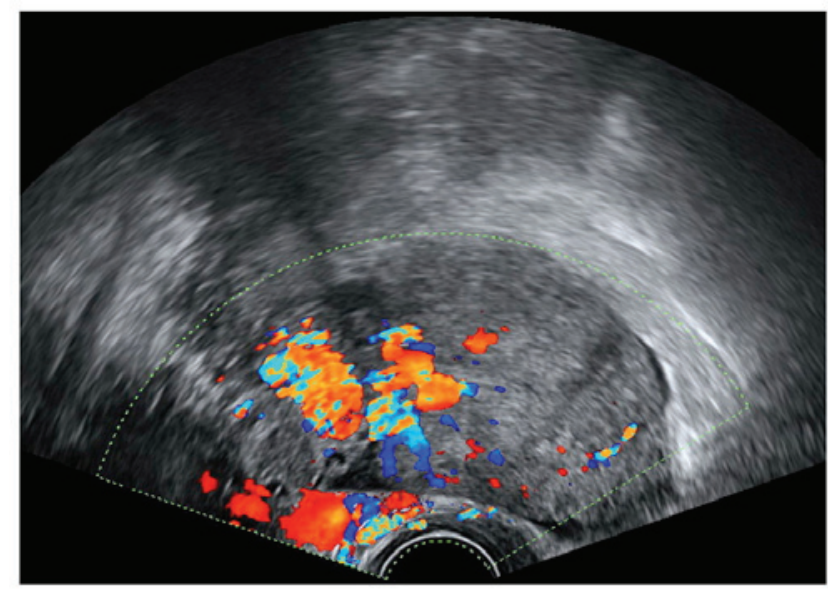

Figure 1. Case 1. Ultrasonography revealed a middle hypoechoic area with a predominantly solid lesion on the left adnexa, with abundant arterial and venous blood flow.

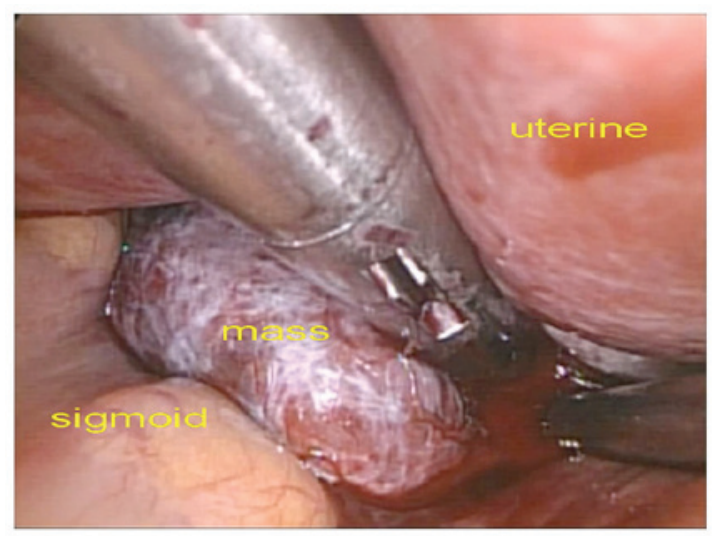

Figure 2. Case 1. During laparoscopy, the left adnexa was identified under the enlarged uterus. The blood surged rapidly from the lesion when clamped.

Case 2. The patient was admitted at the 16th week of gestation, with persistent mild lower abdominal pain. She had undergone in vitro fertilization and embryo transfer and laparoscopic right salpingostomy for ectopic pregnancy with a uterine pregnancy at the 9 th week. A transvaginal ultrasound indicated the presence of an inhomogeneous, predominantly solid lesion, sized $58 \times 56 \times 47 \mathrm{~mm}$, on the left side of the uterus. Doppler ultrasound revealed a blood vessel encircling the mass. The ovaries were of normal morphology and size, with a normal double uterine pregnancy. The ultrasound diagnosis was fallopian tube cancer. The patient exhibited increased CA125 levels $(256.1 \mathrm{U} / \mathrm{ml})$. However, on laparoscopy, we observed an obsolete ectopic pregnancy in the left fallopian tube and salpingectomy was performed. The patient underwent a caesarean section at the 34 th week of gestation as a result of pregnancy-induced hypertension syndrome and the birth weight of the infants was 2,200 and 2,450 g.

Case 3. The patient was admitted during the 20th week of gestation, asymptomatic. Ultrasonography revealed a left-sided $18 \times 16 \times 15-\mathrm{cm}$ adnexal mass, with a heterogeneous echo. The patient had increased CA125 $(532.75 \mathrm{U} / \mathrm{ml})$ and CA199 $(13.87 \mathrm{U} / \mathrm{ml})$ levels. The fetus and the contralateral adnexa appeared to be normal. The mass was diagnosed as ovarian cancer. A left adnexectomy was performed by laparotomy. Frozen section examination revealed a mature teratoma with abundant nervous tissue, which was pathologically confirmed postoperatively. The patient delivered by cesarean laparotomy at 40 weeks and the infant's weight was $3,250 \mathrm{~g}$.

Case 4. The patient was admitted during the 22th week of gestation, asymptomatic. A $15-\mathrm{cm}$ adnexal mixed-echo mass was detected attached to the uterine wall. On Doppler examination, the mass exhibited abundant blood flow and a tangled bloodstream, and was diagnosed as right ovarian cancer. The patient had elevated CA125 levels $(872.7 \mathrm{U} / \mathrm{ml})$. However, the patient refused surgery; she underwent caesarean section at the 39th week and the birth weight of the infant was $3,050 \mathrm{~g}$. Intraoperatively, a $16-\mathrm{cm}$ mass arising from the uterine isthmus was identified, surrounded by dilated blood vessels; the mass was not excised. Both ovaries and fallopian tubes were of normal appearance. After 1 year, the patient was readmitted for the uterine mass. Laparoscopy was performed and a uterine myoma with degeneration was pathologically confirmed.

\section{Discussion}

Although adnexal masses complicating pregnancy are significantly less common compared with non-pregnant patients, benign masses, including benign teratoma (7-37\%), serous cystadenoma (5-28\%) and mucinous cystadenoma (3-24\%), endometrioma $(0.8-27 \%)$, paraovarian cysts $(<5 \%)$ and leiomyoma (1-2.5\%), may be encountered. Ovarian cancers (including those of low malignant potential) in pregnancy are very rare, accounting for $\sim 1-8 \%$ in all adnexal masses (4).

Adnexal masses identified as malignant tumors during pregnancy are a serious finding, due to the increased risk of obstetric complications and the difficulty in surgical management.

Ultrasound evaluation is the usual method applied for the diagnosis of adnexal masses (5). During pregnancy, the ultimate purpose of an ultrasound examination is to assist the gynecologist in determining whether those adnexal masses require observation or surgical intervention.

Characteristics such as septation, solid components, nodules, papillary components, or an average diameter of $>5 \mathrm{~cm}$ have been identified as predictors of malignancy. The 4 benign cases in our study were consistent with the abovementioned findings. It appears to be difficult to distinguish between benign and malignant adnexal lesions during pregnancy. These difficulties are due to the inhomogeneous echo and, particularly, the massively increased pelvic blood flow. DePriest and DeSimone reported that investigations with regard to blood flow on Doppler demonstrated that Doppler examination did not further facilitate diagnosis compared with gray-scale sonography alone, with a similar false-positive rate of $49 \%$ for the prediction of malignancy (6). Groszmann et al did not identify any morphological or vascular flow characteristics that were helpful in sonographically distinguishing benign decidualized endometrioma from malignancy (7).

Undeniably, as a commonly used epithelial ovarian cancer tumor marker, CA125, may be elevated during the first trimester 
of pregnancy and postpartum in normal pregnancy (1). However, massively elevated CA125 levels (1,000-10,000 U/ml) during the second trimester may mislead the gynecologist to suspect malignancy (8). In our study, the CA125 levels were increased above normal levels in all 4 cases.

A limitation of our study was the small sample size. Although the data were from a single center, the results revealed that abundant blood flow and heterogeneous echo of the adnexal masses during pregnancy may not represent malignancy.

\section{Acknowledgements}

The present study was supported by the Science and Technology Commission of Shanghai Municipality (grant no. 134119a8200).

\section{References}

1. Naqvi M and Kaimal A: Adnexal masses in pregnancy. Clin Obstet Gynecol 58: 93-101, 2015.
2. Marret $\mathrm{H}$, Lhommé $\mathrm{C}$, Lecuru $\mathrm{F}$, Canis $\mathrm{M}$, Lévèque J, Golfier $\mathrm{F}$ and Morice P: Guidelines for the management of ovarian cancer during pregnancy. Eur J Obstet Gynecol Reprod Biol 149: 18-21, 2010.

3. Aggarwal P and Kehoe S: Ovarian tumours in pregnancy: A literature review. Eur J Obstet Gynecol Reprod Biol 155: 119-124, 2011.

4. Hoover K and Jenkins TR: Evaluation and management of adnexal mass in pregnancy. Am J Obstet Gynecol 205: 97-102, 2011.

5. Yacobozzi M, Nguyen D and Rakita D: Adnexal masses in pregnancy. Semin Ultrasound CT MR 33: 55-64, 2012.

6. DePriest PD and DeSimone CP: Ultrasound screening for the early detection of ovarian cancer. J Clin Oncol 21 (Suppl): 194s-199s, 2003.

7. Groszmann Y, Howitt BE, Bromley B, Feltmate CM and Benacerraf BR: Decidualized endometrioma masquerading as ovarian cancer in pregnancy. J Ultrasound Med 33: 1909-1915, 2014.

8. Goh W, Bohrer J and Zalud I: Management of the adnexal mass in pregnancy. Curr Opin Obstet Gynecol 26: 49-53, 2014. 